\title{
LE RÔLE SYNTAXIQUE ET PRAGMATIQUE DES CONNECTEURS DANS LE DISCOURS ARGUMENTATIF FRANÇAIS ${ }^{1}$
}

\section{Introduction}

L'objet du présent article sont les connecteurs dans le discours oral français. Les connecteurs en tant que marqueurs phrastiques et interphrastiques assurent la cohérence du discours mais peuvent aussi apparaître comme porteurs de l'argumentation. Les connecteurs en question proviennent de l'analyse du discours oral médiatique français ${ }^{2}:$ le corpus pour l'analyse a été composé de la transcriptions de 6 heures d'enregistrements du discours médiatique français, provenant surtout des tables rondes des émissions Polémiques et Bouillon de culture, enregistrées et transcrites entre 1997 et 1999.

Le terme connecteur dans l'analyse des langues naturelles a été utilisé pour la première fois chez Harris (1970) au sein de la linguistique distributionnelle. On rencontre également ce terme en logique dans le sens du connecteur comme opératuer binaire assurant le lien entre les propositions. Vers la fin des années 70, van Dijk a commencé à délimiter la notion des connecteurs sémantiques et pragmatiques. En même temps en France, $\mathrm{O}$. Ducrot s'est consacré aux études des connecteurs dans le cadre de la pragmatique intégrée à la sémantique. De son oeuvre s'est inspirée l'approche dite genevoise autour de E. Roulet qui s'est centré sur la problématique de l'articulation du discours. J. Moeschler, lui aussi issu de l'école de Genève, s'est aussi inspiré dans la pragmatique de la pertinence, introduite par D. Sperber et D. Wilson en 1986 et qui s'est répandue surtout dans les années 90 . Récemment, l'intérêt pour les connecteurs en France est centré autour de M. A. Morel et l'analyse de l'oral spontané.

Dans les 15 dernières années les connecteurs ne cessent d'interésser les syntacticiens et les pragmaticiens. Nous allons démontrer les résultats de l'analyse des connecteurs les plus fréquemment utilisés dans le discour argumentatif oral.

\section{Les connecteurs entre la syntaxe et la pragmatique}

\subsection{La définition des connecteurs}

En quoi les connecteurs diffèrent-ils des conjonctions? Les conjonctions, dans la grammaire traditionnelle, agissent entre les groupes de mots ou à l'intérieur de la

1 Le présent article est tiré de la thèse du doctorat, défendue en 2000 devant le jury composé des professeurs suivants: prof. Mitja Skubic en tant que président, prof. Vladimir Pogaznik en tant que tuteur, et prof. Janez Justin en tant que membre.

2 Le discours pour nous signifie le texte dans le cotexte de l'énonciation. 
phrase en tant que coordonnants ou subordonnants. Ce sont les termes de l'analyse syntaxique.

Mais la syntaxe, en tant que système formel d'organisation des signes, est instrumentale par rapport à la sémantique, qui s'occupe du sens des signes, et la pragmatique, qui traîte l'organisation des signes dans le contexte.

La définition du terme conjonction ne répond pas à tous les besoins de l'analyse du discours parlé. Ci-dessous, il y a deux exemples où la portée des connecteurs dépasse la phrase, où le connecteur enchaîne directement sur le contexte ${ }^{3}$ (exemple (1)) ou bien sur les énoncés précédents (exemple (2)) :

(1)

C'est pourquoi il n'a pas voulu venir!

(2)

$A:$ Le restaurant est fermé

$B:$ Alors, qu'est-ce qu'on fait?

Pour l'analyse des exemples de ce type, nous sommes obligés d'introduire la notion des connecteurs, qui, d'après la définition de Riegel, Pellat et Rioul (1994 : 617) »sont des éléments de liaison entre des propositions ou des ensembles de propositions ; ils contribuent à la structuration du texte en marquant des relations sémantico-logiques entre les propositions ou entre les séquences qui les composent.

Les connecteurs apparaissent toujours dans les schémas comme celui de l'exemple (3), qui peut être illustré par l'exemple (2) du haut :

(3)

Enoncé. Connecteur, énoncé.

(Dik, $1997: 440$ )

S. C. Dik ajoute que »les connecteurs ne s'identifient pas aux coordonnants, qui ont un fonctionnement inter-phrastique même s'ils sont sémantiquement identiques «. ${ }^{4}$

La définition des connecteurs qui se veut la plus générale ${ }^{5}$ est donnée par J. Moeschler (1998: 77) :

»Un connecteur pragmatique est une marque linguistique, appartenant à des catégories grammaticales variées (conjonctions de coordination, conjonctions de subordination, adverbes, locutions adverbiales), qui :

a) articule des unités linguistiques maximales ou des unités discursives quelconques

b) donne des instructions sur la manière de relier ces unités

3 Le contexte possible serait une surprise-partie que le fils organise pendant l'absence des parents et l'énoncé de l'exemple pourrait être attribué à ces mêmes parents au moment de l'arrivée à la maison.

4 A l'original: Connectives are not to be equated with coordinators, which function intra-clausally, even if they are semantically identical.

5 Moeschler dit (1998: 77) »Voici donc la définition la plus générale et la plus consensuele possible que l'on peut donner des connecteurs pragmatiques.« 
c) impose de tirer de la connexion discursive des conclusions qui ne seraient pas tirées en son absence.«

Les conclusions les plus importantes que nous pouvons tirer de ces définitions pour notre recherche sont les suivantes :

1 - les connecteurs font partie de l'analyse du discours

2 - les connecteurs sont polysémiques, agissant au niveau sémantique ou pragmatique.

\subsection{Les types des connecteurs}

D'après Roulet et al. (1985: 85-193), les connecteurs appartiennent aux types suivants : les marqueurs de fonction illocutoire, les marqueurs de fonction interactive et les marqueurs de structuration de la conversation.

Les marqueurs de la fonction illocutoire sont initiatifs et réactifs du type suivant (exemple (5)) :

(5)

J'aimerais vous demander quelque chose : quel type de relation avez-vous avec elle, au juste?

(Roulet et al., $1985: 86$ )

Le marqueur initiatif $j$ 'aimerais vous demander quelque chose a, comme d'ailleurs aussi les autres marqueurs du même type, une construction phrastique mais est fonctionnellement équivalent à un connecteur.

Les marqueurs de la structuration de conversation apparaissent surtout dans le discours oral : d'un côté, ils sont dotés de la faculte d'organiser le discours en unités tout en signalant la cohésion des unités et les possibilités de l'ouverture ainsi que de la clôture des thèmes (exemple (6)) :

(6)

MC : Alors, tout cela, vous êtes conquis par les propositions du ministre ou vous les contestez donc, peut-être rappelez-les, Monsieur Collin.

(Corpus 2/2)

Les connecteurs les plus typiques et les plus largement répandus sont les marqueurs de fonction interactive. Ils déterminent le rapport entre les constituants d'une intervention (Roulet et al., $1985: 111$ ) et se distinguent d'après leurs fonctions syntaxiques et pragmatiques. Au niveau pragmatique, nous distinguons trois classes des connecteurs interactifs selon les types de relations entre les énoncés à l'interieur d'une intervention:

1) les connecteurs argumentatifs

si la relation est exprimée sur le constituant subordonné c'est la relation causale, introduite par des connecteurs car, parce que, en effet, d'ailleurs, puisque. Si la relation s'exprime sur le constituant directeur, c'est la relation consécutive, introduite par les connecteurs donc, alors, par conséquent, aussi. 
2) les connecteurs contre-argumentatifs introduisent la relation du contre-argument à l'acte directeur ; ce sont mais, bien que, quand même.

3) les connecteurs conclusifs

servent à l'expression de la conslusion : finalement, au fond.

\subsection{Les corrélations entre les caractéristiques syntaxiques et pragmatiques}

Selon les définitions ci-dessus, les connecteurs agissent au sein de la pragmatique parce qu'ils dépassent les limites de la phrase. Ils appartiennent aux différentes classes syntaxiques, mais aucune des classes n'est constituée exclusivement des connecteurs. Syntaxiquement, les connecteurs sont rassemblés dans les classes syntaxiques ou les parties du discours suivantes : les coordonnants (mais, et, car), les subordonnants (parce que, puisque), les adverbes (alors, finalement).

Les connecteurs pragmatiques agissent sur les différents niveaux syntaxiques : soit entre les propositions, soit entre les énoncés mais aussi au niveau de l'énonciation. Nous allons en parler au cours de l'analyse des connecteurs les plus fréquents.

\section{Les connecteurs les plus fréquents du corpus analysé et leur rôle dans le discours}

Nous allons démontrer les valeurs et les fonctions des connecteurs et, parce que, alors, donc, mais qui ont été le plus fréquemment utilisés dans le discours analysé. Les connecteurs car, puisque, d'ailleurs, quand même, c'est-à-dire et enfin qui, à leur tour faisaient aussi l'objet de l'analyse, ne seront pas traîtés ici.

\subsection{Et entre la conjonction et le connecteur}

En tant que conjonction, et unit les éléments de la même nature, les groupes nominaux ou les adjectifs (exemples (7) et (8)) ou de la même fonction d'épithète (exemple (9)) :

Les phénomènes d'emploi, le phénomène de l'aménagement du territoire, et aussi tous les impéraififs du développement...

(Corpus 2/30)

(8)

Cela faisait rêver bien de filières artisanales et industrielles qui, dès qu'ils ont une crise ou un problème ... ou un produit qui ne se vend pas demanderaient à l'Etat...

Un paletot court et sans manches

Si l'élément introduit par et n'appartient pas à la classe des noms de la même nature ou fonction, il se produit l'effet de syllèpse (exemples sous (10), les deux sont tirés de Morel, 1998) : 
(10)

Il est venu avec son enthousiasme et sa valise.

Il a pris le métro et son temps.

Au niveau de la proposition ou de l'énoncé, et peut aussi jouer le rôle du connecteur pragmatique. Dans ce cas-là, et $\mathbf{n}$ 'indique pas que deux éléments linguistiques agissent au même niveau ou ont le même statut syntaxique, mais présente toujours la hiérarchie parmi les éléments discursifs. Par cela, il souligne soit la complémentarite du second élément en relation du premier soit recatégorise le discours précédent de manière d'en faire la base de ce qui va suivre (exemples sous (11)) :

(11)

Il savait tout, et depuis longtemps.

J'en avais, et beaucoup.

(Morel, 1998)

$E t$ marque le changement du niveau discursif et du plan énonciatif : X est donné comme partagé par l'interlocuteur et pris en compte dans la coénonciation ${ }^{6}$ (c'est la pratie après PG4 dans notre exemple (12)), tandis que $Y$ (après PG5 dans le même exemple) n'est pas l'objet de la coénonciation. Seul le locuteur, qui recentre l'énonciation sur lui-même, en est responsable. Habituellement, l'assertion introduite après et commence par je :

(12)

PG4 : J'ai la même opinion qu'Alexandre Adler sur le juge Gazone, ... en fait je connais bien ce dossier..

MC35 : Vous permettez...

PG5 : Et j'ai aussi des arguments supplémentaires à apporter, à apporter à l'appui de sa thèse. Mais reste que la dictature chillienne, comme d'ailleurs la distature argentine ou la dictature uruguayenne et la dictature brésilienne ont tué de façon parfaitement criminelle des ressortissants de nos pays...

(Corpus 6)

Les autres fonctions de base du connecteur et sont les suivantes (d'après M. A. Morel, 1998) :

Et marque la complémentarité des éléments hétérogènes au niveau des faits ou bien au niveau des types d'opération (exemple (13)) :

(13)

C'est la production qui est gérée par la météo, la météo gère à la fois des sorties et à la fois la consommation. Et effectivement, les surproductions qui arrivent toute l'année ne sont(soient) que ponctuelles.

(Corpus 7/3)

6 Coénonciation ou la prise en compte du co-locuteur (M. A. Morel, 1998). 
Et marque le procès d'addition. L'exemple (14) montre l'addition des faits à un ensemble créé au discours, et dans sa dernière occurrence, et est renforcé par puis :

PC $13:$ C'est un livre / c'est un livre en miroir. Et le titre est en miroir. Et c'est cette espèce d'envie qu'ont sou... je pense, qu'ont souvent les femmes, d'abord, de se regarder dans l'autre, de se voir d'abord physsiquement et puis de se voir intellectuellement.

(Corpus 3/28)

$E t$ assure la relation avec le contexte situationnel et globalise ce qui a été dit. Le connecteur et introduit le fait qui a le statut de rhème par rapport au reste qui devient le thème (exemple (15)).

Je vous renvoie, mon ami Alexandre Adler, à la convention internationale contre la torturel qui a été ratifiée par l'Espagne et par l'Angleterre, eh ben, et cette convention que dit-elle que lorsque les personnes sont supposées et présumées avoir commis des actes de torture elles doivent être soit jugées soit éventuellement extradées sur territoire de l'Etat sur lequel elles se trouvent.

(Corpus 6/43)

$E t$ introduit le commentaire sur les faits du discours, soit sur la situation spécifique du discours et du monde cognitif que le locuteur partage avec le collocuteur :

(16)

Je pense qu'il faut/si l'on envisage, et je crois qu'il faut l'envisager puisqu'à l'hôpital par exemple on l'envisage, le construire avec les partenaires, et sans doute pas au milieu d'un conflit.

(Corpus 7)

$E t$ dans l'exemple (16) marque la consensualité sur le sujet du discours, ce qui explique l'emploi de ce connecteur au début des incises. Le connecteur et enchaîne sur la situation discursive, parfois même avec l'emploi cataphorique.

Et peut apparaitre aussi dans le rôle du connecteur textuel ; il introduit la connexion parmi les éléments où il n'y a aucun lien logique apparent. Il ne s'agit pas de la consécution temporelle; et assure la cohésion parmi différentes épisodes du récit dans un texte cohérent. Les faits et les événements deviennent complémentaires dans la conscience de l'allocutaire grâce au connecteur et (exemple (17)) :

$S G$ : Elle n'aura de cesse de l'attendre/surtout, finalement puisque elle ne sait même plus chercher. Et cette vieille femme qui était tellement habituéelétait une sorte de tombeau vivant, mais il est évident que le vrai tombeau c'est dans les survivants, et puis les autres tombeaux, c'est... c'est symbolique. (...) Et cet enfant donc sera élevé jusqu'à l'âge de 11 ans/par cette vieille femme qui porte en plus plusieurs langues et plusieurs cultures en elle.

(Corpus 3/244) 
Dans le corpus analysé apparaît aussi le et métadiscursif des énoncés qui servent du commentaire aux énoncés précédents (exemple (18)) :

(18)

BP146: La veille de ses cent ans et là je dois lire une page, alors je me mettais à lire, $j$ 'ai trouvé ce passage très beau, par exemple, donc, on l'enterre, elle, là, on l'enterre...

(Corpus 3/235)

Dans le corpus analysé, et avec ses 695 occurrences apparaît comme la conjonction et le connecteur le plus fréquemment utilisé - dans 404 exemples, il joue le rôle de conjonction unissant les éléments de la même nature ou fonction, ajoutant des information complémentaires, tandis que dans les autres 281 occurrences, et est le connecteur au niveau des énoncés où il garde sa valeur de complémentarité mais au niveau des éléments hétérogènes. $E t$ thématise le donné et rhématise ce qui suit. Dans ce cadre, nous pouvons expliquer ses différents emplois, illustrés ci-dessus : le lien avec le contexte, l'introduction du commentaire, l'empoi métadiscursif. Et en tant que connecteur introduit la consensualité (Morel, 1998) et se place dans le cadre plus vaste de la coénonciation.

\subsection{Parce que - le connecteur argumentatif et le marqueur de l'énonciation}

Parce que est le connecteur argumentatif le plus fréquemment employé dans le corpus analysé. Du point de vue syntaxique, c'est la conjonction qui donne la réponse à la question pourquoi?. D'après M. A. Morel (1998), parce que permet d'introduire une cause factuelle ou le motif d'un procès. La subordonnée introduite par parce que a le statut du rhème, puisqu'elle introduit une information nouvelle que le locuteur ne partage pas avec le co-locuteur.

Les valeurs causales de parce que qui agit au niveau de la proposition ont été largement démontrées. Dans ces emplois, parce que connecteur ne diffère pas de parce que conjonction. Pour démontrer les valeurs spécialisées de parce que connecteur, nous allons nous limiter à son emploi au niveau des énoncés : parce que dans l'exemple (19) enchaîne sur l'énonciation, ce qui se voit de sa paraphrase (19') (les deux exemples sont tirés de Moeschler (1994: 47)) :

(19)

Il y a du poulet au frigo parce que je n'ai pas envie de faire à manger.

Je dis qu'il y a du poulet dans le frigo parce que je n'ai pas envie de faire à manger.

Dans le corpus analysé il y avait 9 exemples de ce genre (sur 165 exemples de parce que au total), parmi lesquels aussi l'exemple (20) ci-dessous il s'agit de la justification de l'énonciation et du parce que métalinguistique : 
(20)

JFKI : D'abord, on revient d'abord à 81 quand on a régularisé/il est arrivé dans les mois qui suivent/... les statistiques sont là ... plus de gens qu'on a régularisé, j'ajoute que Noël Mamère dont je dis d'ailleurs, parce qu'il faut que les téléspectateurs le sachent, on va peut-être avoir un affrontement dur, on est amis.

(Corpus 5/1)

Parce que peut aussi réintroduire le thème au début de l'énoncé :

(21)

GC31: C'est pas mon débat, eh ben, on aurait pu parler de la différence qui existe entre le dopage et le traitement de la douleur chez le sportif.

BK103 : Bien sûr.

GC32: Parce qu'il y a souvent la confusion à ce qui est le traitement de la douleur et à ce qui est le dopage.

(Corpus 7/24)

Le connectuer parce que apparaît très rarement au début des énoncés. Les exemples de ce type font plutôt l'exception à la règle puisque parce que enchaîne sur les énoncés précédents ou le contexte. Parce que dans ces cas-là peut être défini en tant que marqueur de la prise de parole (Berthoud : 1996).

Parce que introduit le jugement sur l'énonciation:

Le connecteur parce que peut marquer le jugement sur l'énonciation, ce qui montre l'exemple (22). Dans cet exemple, parce que joue le rôle métadiscursif :

BP 139 : Très bien. Merci. Alors, on va terminer par Tobie des Marais de Sylvie Germain'et je crois qu'on va terminer en beauté parce que c'est vrai, il faut le répéter, je trouve ça un roman d'une très grande beauté violente et noire qui plonge ses racines dans le ciel et dans les têtes se trouve dans le ... dans les marais ... poitevins.

(Corpus 3/37)

Des 165 exemples de l'emploi de parce que, 101 occurrences du corpus servaient pour la justification des faits ou des idées, il y avait 9 cas de la justification de l'énonciation des faits, 24 exemples de la réintroduction du thème, 13 exemples où l'énonciation introduite par parce que n'est pas terminée et 4 exemples où parce que n'exprimait pas la cause.

\subsection{Alors}

Alors est un connecteur consécutif. A l'origine, alors avait la valeur temporelle et a gardé sa valeur d'anaphorique. Dans le corpus analysé, seulement deux occurrences d'alors (de 175 au total) avaient la valeur temporelle

Alors marque la conséquence (exemple (23) ci-dessous :

(23)

$G C$ : Vous n'êtes pas accusé. 
BK: J'ai le sentiment de l'être, alors si vous permettez, s'il vous plait, j'ai envie de répondre.

(Corpus 7/19)

Alors en tant que marqueur de structuration discursive est apparu 133 fois sur 175 occurrences de alors en total (exemple (24)) :

MC : ...P.H., numéro deux, je crois qu'on peut le dire, de l'équipe du Festina, accompagné de son avocat, maitre Gilbert Collard. Alors, avant de nous lancer sur la santé, Bernard Kouchner, quelques questions plus politiques vous posez à poser au gouvernement Jospin où vous êtes...

(Corpus 7/1)

Le connecteur alors dans l'exemple (24) n'introduit pas une consequence mais indique simplement que la modératrice $\mathrm{MC}$ va ouvrir un nouveau thème. Alors est dans ce cas-là le marqueur de structuration discursive, ce qui a été découvert par $\mathrm{E}$. Roulet et ses collaborateurs (1985), et M. A. Gerecht (1987). Ce alors agit au niveau de l'énonciation. Dans notre corpus, nous avons aussi découvert alors métalinguistique qui introduit le commentaire.

Alors en tant que marqueur de la structuration discursive peut apparaître au début (exemple 25) ou au milieu de l'intervention (exemple (26)) : alors de l'exemple (25) a pris l'intonation interrogative :

(25)

MC : Alors, Messieurs les politiques, ça, vous y êtes d'accord?

(Corpus 2/4)

Lorsque l'énonce où figure alors prend l'intonation interrogative, nous pouvons le présenter comme s'inscrivant dans un univers commun aux interlocuteurs. Il construit une représentation commune au locuteur et au co-locuteur sur laquelle il enchaîne et situe par consequent l'énoncé du côté de la consensualité.

Dans les exemples 24 et 25 , alors est situé au début de l'intervention (et du paragraphe selon M. A. Morel). M. A. Morel dans son schéma de la coénonciation dit à propos de alors que "alors en intonation légèrement montante à la finale, introduit un fait conforme aux attentes qu'implique la thématique générale développée. A ce titre il peut introduire un fait qui permet de boucler une sequence événementielle. Alors suppose ainsi une attitude consensuelle et la convergence obligée des points de vue sur la correlation des événements" (1998: 115). Cette explication est valable pour notre exemple (26):

(26)

BP : Mais c'est bien. Eh bien, on va passer au roman de Françoise Chandernagor. Est-ce qu'il est aussi facile de parler d'un roman comme celui-ci à la télévision alors que vos précédents étaient des romans historiques. Alors, celui-ci évidemment, c'est un roman à la charge d'aujourd'hui.

(Corpus 3/20) 
V. Traverso (1998 : 135-136) classe alors parmi les marqueurs d'ouverture de theme, parmi lesquels figurent aussi tiens et $t$ 'sais. Ces marqueurs sont utilisés pour indiquer la coherence thématique. Alors-marqueur de structuration de conversation apparaît dans ce rôle puisque les moderateurs s'en servent pour poser de nouvelles questions.

Alors peut aussi inclure l'énoncé dans le thème de la conversation (exemple (27)) que les digressions ont efface ou bien marquer la structuration des sous-thèmes par rapport à un thème central (exemple (28)) :

Mais je crois que le Nord est presque installé au niveau commercialisation sur un modèle breton et, enfin, c'est ce que j'appelle plutôt le modèle gouverné, il a, ces gens alors ont une méthode de gouvernement en matière de politique légumière qui est scandaleuse et qu'il faudrait bien faire rentrer en raison. Nous, on souhaite un Etat qui prenne sa place, qui arrête de gérer le rapport de force et qui ait un projet véritable pour l'agriculture.

(Corpus 2/3)

On parle de plus de 50000 d'entreprises de production, on parle de 20 milliards de chiffres d'affaires au niveau de la production et on parle de 35 légumes. Alors, aujourd'hui, aujourd'hui depuis que nous posons les questions au ministre le 25 juillet, le 25 novembre, le 6 avril et puis des séries de rencontres, on nous répond au niveau du Ministère, tout à coup on nous répond "choux-fleurs : Bretagne". Je rappelle: il y a 35 légumes, il y a une trentaine de départements concernés. (Corpus 2/1)

La fonction de la cohésion discursive, assurée par alors, est liée à sa valeur anaphorique. Dans tous les énoncés présentés, alors marqueur de structuration discursive introduit une nouvelle étape du mouvement discursif tout en signalant que cette nouveauté s'inscrit dans le cadre thématique consensuel des co-locuteurs. Les occurrences de alors sont les pivots du discours en formation, de son développement et sa cohésion même si elle ne vient que des idées et des associations du locuteur.

Alors métalinguistique introduit les énoncés qui portent sur le dit, qui introduisent une explication ou un commentaire. Dans le corpus analysé, il y avait 10 exemples de ce type parmi lesquels l'exemple (29), où l'énoncé enchaîne sur l'énonciation et la demande de la permission de l'énonciation :

BK : Bien sûr, aux représentants élus de la proximité, c'est le rôle des associations aussi dans la vie politique. Tout ça n'est pas une déclaration de guerre, au contraire. Puis alors permettez-moi de vous dire que n'étant ni Giscard d'Estaing ni Jacques Chirac, je ne peux répondre à leur place.

(Corpus 7/2) 
Dans le corpus analysé, nous avons dénombré 175 occurrences de alors, dont 10 de alors temporel, 32 de l'emploi consécutif, et 133 occurrences de alors marqueur de structuration discursive (dont 16 fois comme alors interrogatif et 10 fois comme métalinguistique). Alors au niveau discursif entre en relation avec d'autres connecteurs dans les combinaisons suivantes : et puis alors, et alors, et donc alors, mais alors et les marqueurs modaux (alors finalement, alors en effet, alors effectivement, alors évidemment, alors justement, alors c'est vrai).

L'emploi de alors est plus typique pour l'émission Bouillon de culture que pour les Polémiques. Dans l'émission Bouillon de culture, l'atmosphère est du côté de la consensualité tandis que dans les Polémiques, nous nous trouvons parfois au milieu d'un conflit. En conséquence, alors est beaucoup moins fréquent ou même absent au moment des confrontations. Nous pouvons conclure que même malgré le glissement du sens du connecteur temporel à travers le connecteur consécutif jusqu'à sa fonction du marqueur du discours alors a gardé une de ses caractéristiques, la consensualité.

\subsection{Donc}

Donc est mobile à l'intérieur de l'énoncé et en plus il est combinable avec d'autres conjonctions et adverbes, p. ex. et donc, donc alors. Dans cette perspective son statut syntaxique de la conjonction de coordination se révèle problématique. Il serait mieux de parler d'un adverbe de relation (Riegel, Pellat, Rioul, 1994 : 525).

Donc est le marqueur de l'inférence spécialisé dans l'introduction de la conclusion d'un raisonnement comme le syllogisme ou le raisonnement mathématique ou encore le discours scientifique. C'est un connecteur consécutif qui exprime que deux faits sont toujours donnés en même temps. C'est ce qui permet en se fondant sur la concomitance d'inférer un fait d'un autre fait (C. Hybertie, $1996: 8$ ), comme c'est le cas dans l'exemple (30) :

Il a des moustaches, donc c'est un gradé.

La relation consécutive opérée par donc dans l'énonciation en cours repose sur une relation plus générale, validée hors du discours. C'est pourquoi l'emploi de donc n'indique pas seulement qu'un fait $\mathrm{x}$ a entraîné un autre fait $\mathrm{y}$ mais aussi que $\mathrm{x}$ et $\mathrm{y}$ sont toujours donnés simultanément. Cela nous permet d'inférer un fait d'un autre fait comme dans l'exemple (30). Ce procédé est possible aussi avec alors, mais là la concomitance n'est pas de règle. La relation consécutive introduite par donc est basée sur une relation plus générale, validée hors du discours. Par le biais de la relation de concomitance qu'il exprime, donc sert à légitimer la validation. La concomitance le met du côté de la consensualité co-énonciative (Morel, Danon-Boileau : 1998). Donc impose la prise en charge de la relation par le co-énonciateur et ce co-énonciateur peut même être élargi à un auditoire universel. 


\subsubsection{Donc en tant que marqueur de structuration du discours}

Lorsqu'il marque la relation de l'identification entre le terme conséquent et le terme antécédent, donc sert à structurer le discours tout en introduisant la reprise ou la récapitulation de l'antécédent par conséquent ou bien en assurant la fonction métadiscursive.

Il peut marquer la reprise ou la récapitulation : en fonction assurant cette fonction, donc ne reprend pas l'antécédent au sens du pronom, mais introduit un terme qui constitue soit une reprise soit une récapitulation du terme antécédent (Hybertie, $1996: 9$ ).

Dans l'exemple (31) ci-dessous, le pronom là reprend l'énoncé précédent (c'est le fait que sur les 503 milliards de soutien publique que de la France reçoit l'Europe à peine 3 viennent pour organiser, pour soutenir la production des fruits et des légumes en France). Donc marque à la fois le lien de dépendance entre la conséquence et le terme antécédent et permet le retour du discours au thème qui a été légerement effacé par les digressions. La capacité de donc d'introduire la reprise ou la récapitulation du terme qui a été énoncé auparavant est baséé sutout sur le fait que l'énoncé introduit par donc présuppose la consensualité, ce qui est l'effet de la simultanéité dans le fonctionnement.

(...) nous avançons véritablement que les problèmes sociaux soient traités et il restera un beau problème à traiter, c'est le fait que sur les 503 milliards de soutien public que la France reçoit de l'Europe à peine 3 viennent pour organiser, pour soutenir la production des fruits et des légumes en France. Il y a donc là des questions qui doivent être traitées et à mon sens on devra les aborder dans la voie de l'orientation agricole et de la réforme parce que les... des légumiers sont aussi les gens qui contribuent à l'emploi.

(Corpus 2/12)

La valeur de l'identification introduite par donc peut prendre la nuance conclusive comme c'est le cas dans l'exemple (32):

BP21 : Et puis alors la fille de Léonore donc c'est la troisième, s'appelle Olivia, elle devient un écrivain public, là aussi, elle est aussi dans une imposture parce qu'elle écrit aux autres à la demande des lettres de rupture, d'amour et là aussi... (Corpus 3/5)

PC6 : Ah oui, elle a commencé très tôt... Et puis il y a l'alter égo de Gloria Pater, peut-être ma préférée, Babette Cohen, qui est une juive, pied noir rapatriée d'Algérie, qui déteste la France et qui s'est faite Américaine et qui a eu un magnifique trajet américain, puisqu'elle a épousé l'homme qu'il fallait aux Etats-Unis, l'homme qui l'a faite Américaine, et puis malheureusement elle a été plaquée huit jours avant... 
BP40 : A 25 ans, d'accord.. Donc toutes ces quatre vont parler à huis clos et c'est que ces quatre femmes, quatre intellectuelles même si la comédienne le moins... (Corpus 3/8)

Donc des exemples (32) et (33) comprend la nuance conclusive, mais il y a une différence dans les valeurs d'un et de l'autre : dans l'exemple (32) donc introduit la proposition qui sert de l'identification (Léonore c'est donc la troisième), tandis que dans l'exemple (33) il s'agit de la différenciation : donc toutes ces quatre présente la rupture et introduit un nouveau thème qui est à la fois le résumé de tous les thèmes mentionnés.

La fonction métadiscursive est aussi liée à la valeur de l'identification que donc peut avoir et qui permet aussi d'introduire le commentaire d'un terme ou d'un énoncé antérieur, car il y a effectivement une certaine identité entre ce que commente et ce qui est commenté. Cette fonction métadiscursive peut avoir des formes différentes, soit comme une construction d'une équivalence sémantico-référentielle entre les termes mis en relation, comme approximatif donc compréhensible (Hybertie, idem, p. 12.), soit comme commentaire de l'énonciation (exemple (34)) où donc métadiscursif introduit le commentaire à l'énonciation du co-locuteur :

JS : Je ne peux pas rentrer dans votre sens. Aujourd hui la production française satisfait à peine autour de $70 \%$ de la consommation française. En Europe, la production des fruits ou des légumes et des fruits est liée à la même problématique pour voir : Europe a à peine 40\%. L'Europe est le premier importateur mondial des fruits et des légumes au rapport du SENAT. Donc je veux dire dans ces chiffres-là qu'il faut intégrer, il n'y a pas de surproduction. Par contre, il y a des téléscopages effectivement, et le cas des choux-fleurs est un téléscopage.

(Corpus 2/7)

Le commentaire discursif de l'exemple (35) est le commentaire de l'énonciation dans l'énonciation dans sa construction polyphonique où le locuteur lit le roman et donne le commentaire :

$B P$ : Après trois ou quatre ans de mariage, un jour comme je rentrais, donc c'est notre romancière qui raconte ça, un jour comme je rentrais d'un séminaire il me disait "Anne est venue à la maison". Anne c'est bon une petite... "Et franchement je te dis qu'elle a trouvé notre intérieur négligé, le ménage mal fait, notre chambre surtout, les draps mal bordés, la table de chevet comblée de poussière... Donc elle découvre d'un seul coup que sa maîtresse lui bouge dans le lit conjugal ...

(Corpus 3/14)

Ainsi en marquant le lien de dépendance du terme conséquent par rapport au terme antécédent et en marquant en même temps leur équivalence, donc permet d'assurer la structuration du discours : recentrage du discours en introduisant une reprise ou une récapitulation. 


\subsubsection{Donc avec la valeur de différenciation : la relation cause - conséquence}

Donc avec la valeur de la différenciation marque la relation entre la cause et la conséquence ; selon C. Hybertie (1996 : 13) il ne s'agit plus de l'équivalence mais de la différenciation de trois types :

1. les conséquences factuelles où l'état des choses décrites dans l'énoncé représente la conséquence du fait de l'énoncé précédent (exemple (36)) où l'énonciateur introduit à l'aide de donc sa propre inférence et opinion concernant l'état des choses :

FC : Mais dans notre génération être fidèle paraissait assez ridicule et donc possessif. (Corpus 3/13)

Avec donc, le locuteur présente son propre énoncé comme une opinion partagée dans l'auditoire, même si ce n'est pas le cas. Il s'agit alors d'un coup de force argumentatif.

2. Le fait qui est inféré à partir des énoncés précédents ou l'état des choses n'est pas donné dans l'expérience du locuteur :

Donc Catherine est sûrement faite pour la fidélité mais elle aime cet homme donc elle accepte tout ça et c'est pas très grave, elle est d'abord la première épouse mais effectivement au sens du harem, c'est-à-dire il y a eu d'autres un peu, là, des combinaisons de passage, mais elle sait qu'elle est la plus importante.

(Corpus 3/13)

Donc, qui cumule l'expression d'une relation de consécution et une relation de concomitance, apparaît comme le marqueur privilégié de l'inférence, qui consiste à remonté d'un fait donné dans l'expérience de l'énonciateur à un autre, non donné dans son expérience.

3. Donc introduit la conclusion qui va clore le mouvement discursif conclusif composé des différents moments d'un raisonnement antérieur : c'est donc de la logique discursive : les idées $x, y$ et $z$ entraînent la conclusion $c$ (exemple (38)) :

Non, le Front National d'abord ne recule pas si on reprend la la la législative de Nice, nous avons progressé de 25,5 de 22,5 à 25, 5, on a même progressé de deux points, donc ce n'est pas du tout un recul.

MC: Mais la gauche a plus progressé donc vous êtes en 3è position au lieu de $2 e$.

Le premier et le deuxième donc de l'exemple (38) marquent le raisonnement logique qui est renforcé avec les chiffres. Les deux mettent les conclusions dans le champ de la co-énonciation, typique pour donc. 


\subsubsection{L'énoncé marqué par donc comporte un parcours}

D'une manière générale, selon C. Hybertie (1996 : 17), un énoncé comporte un parcours lorsqu'on ne peut pas ou on ne veut pas assigner une valeur référentielle définie à l'un des éléments de cet énoncé. Il y a alors parcours sur la classe de toutes les valeurs assignables à cet élément. Sans qu'il soit possible d'en distinguer une seule. C'est le cas, en particulier de l'interrogation, de l'injonction et de l'exclamation dont nous avons découvert quelques exemples dans notre corpus analysé (exemples (39), (40) et (41) :

BP22 : Pourquoi avez-vous choisi donc ces femmes qui sont justement dans une sorte de, justement, de mensonge, d'équivoque?

(Corpus 3/6)

Ces femmes de l'exemple (39) ci-dessus, introduites à l'aide de donc, forment un ensemble par rapport aux autres femmes. Dans l'exemple (40), pourquoi donc est énoncé avec une nuance d'impatience parce qu'il est évident que le locuteur cherche la réponse parmi toutes les occurrences possibles des réponses. Dans les deux cas il s'agit des questions partielles.

GM: Alors justement, je vous ai entendu, je vous avais entendu, il y a quelques minutes, parler de la construction européenne. Pourquoi les gens de la SNCF ou d'autres font-ils grève en ce moment. Enfin je veux dire les dernières grèves. Pas pour des revendications qui étaient recurielles... pas pour.. pas pour.. /

$M C$ : Pourquoi donc?/ pas pour une diminution de la durée du travail /

$C D$ : Essentiellement pour des hommes.../ Mais pour l'amélioration du service public.

(Corpus 8/13)

L'exemple où donc introduit un énoncé impératif (41). Donc introduit la conclusion logique et en même temps exprime l'impatience du locuteur envers le co-locuteur:

$C J$ : Rappelons quand même que dans le $C M$, dans l'organisation commune de marché des fruits et légumes, c'est le seul secteur où les producteurs s'en contribuent, c'est-à-dire, quand on verse $1 F$ lorsque les producteurs cotisent pour $1 F$, Bruxelles reverse $2 F$. Ça n'est pas contribuable français, ce sont les producteurs. /MHA : C'est l'argent public/ Non, d'accord, mais ce sont des producteurs. Donc ne dites pas...

(Corpus 2/8)

Donc avec sa valeur d'identification est susceptible d'exprimer soit la structuration du discours soit la différenciation s'il s'agit du mouvement argumentatif d'inférence. Les valeurs dénombrées dans le corpus analysé étaient les suivantes : il y avait 132 occur- 
rences de donc, dont au niveau discursif : $55 \%$ de toutes les occurrences ( 72 exemples, dont 45 marqueurs de la structuration discursive et 27 emplois métadiscursifs).

Donc introduisant la conséquence est apparu 60 fois dont 17 occurrences de la conséquence factuelle, 26 exemples d'inférence et la conséquence logique dans l'énoncé déclaratif (13), interrogatif (3) ou impératif (1).

L'analyse du corpus a montré que les deux fonctions de donc, la fonction discursive et la fonction argumentative sont balancées. Le locuteur a en effet de faire l'inférence pas seulement à la base des faits, mais surtout à la base du raisonnement. Les exemples impliquant le raisonnement sont beaucoup plus nombreux des"exemples d'inférence factuelle.

\subsection{Mais - connecteur contre-argumentatif}

Mais dans son rôle de connecteur contre-argumentatif introduit un contre-argument, mais son fonctionnement est loin d'être unifié. Ducrot (Ducrot et al., 1980 : 93- 131) en a découvert deux valeurs : mais de réfutation et mais d'argumentation où il s'est appuyé sur les éléments extra-linguistiques comme le contexte, les intentions conversationnelles ainsi que les jugements des locuteurs envers la situation et leurs relations.

D'après Ducrot (Ducrot et al., $1980: 98$ ), le connecteur mais n'indique pas à proprement parler que $P$ et $Q$ sont deux informations opposées en elles-mêmes : elles ne s'opposent que par rapport à un mouvement argumentatif mis en évidence par la conclusion $r$. En plus, mais nécessite une référence à la situation d'énonciation, car la conclusion $r$ qui sert de lien entre $P$ et $Q$ n'est pas seulement déterminée par le contenu de ces deux propositions, mais dépend pour une bonne part des croyances des interlocuteurs.

Ayant pris en considération ses recherches aussi bien que les conclusion plus récentes de Jean-Michel Adam (1990:191-226), nous avons découvert et décrit 5 valeurs de mais qui apparaissent dans le corpus analysé : mais de renforcement-rencherissement, mais de réfutation, mais phatique, mais concessif et mais argumentatif.

\subsubsection{Mais de renforcement-rencherissement}

Ce mais apparait dans le corpus 5 fois (de 323 occurrences de mais en total) sous forme non seulement dans la proposition P... mais même, aussi, également, en plus, de plus dans la proposition $\mathrm{Q}$ et en tant que tel apporte un argument additionnel pour la conclusion $r$. P est habituellement connu, avec la valeur du thème et $Q$ nous donne le rhème. Dans les deux exemples tirés du corpus (exemples (42), (43)), mais indique que $P$ est donné et $\mathrm{Q}$ nouveau :

(42)

$M C$ : Vous avez considéré que c'est une intrusion sur l'avis politique ou alors? $B M$ : Je considère d'une part que le le le message et le programme du Front National est parfaitement compatible avec les enseignements de l'église et que l'attitude de cet évêque est non seulement indigne aux regards des règles. de l'église 
mais est tout à fait scandaleuse dans les débats politiques français.

(Corpus 6/10)

(43)

MC : Pascal Hervé, Pascal Hervé, vous faites ça, vous, pas seulement l'altitude, mais aussi...

PH : Mais non, on s'en remet à nos médecins, à un médecin de sport hein, et c'est peut-être ce jour-là...

(Corpus 7/51)

Dans l'exemple (42) les arguments pour la conclusion le rapport de l'église envers le FNn'est pas juste sont P (l'attitude de cet évêque est non seulement indigne aux regards des règles de l'église) et $\mathrm{Q}$ (mais est tout à fait scandaleuse dans les débats politiques français). L'argument $\mathrm{Q}$ est la gradation de ce qui a été donné en $\mathrm{P}$ et il est impossible d'inverser l'ordre des propositions.

Dans l'exemple (43), le point de départ pour la proposition $Q$ est donné dans l'énoncé de la modératrice $\mathrm{MC}$, on s'attend à un argument plus fort que $P$ (pas seulement l'altitude). Mais le locuteur PH introduit un nouveau énoncé par mais non avec lequel il réfute l'énonciation de la modératrice.

\subsubsection{Mais de réfutation}

Mais de réfutation se combine avec $\mathrm{P}$ à la forme négative, qui peut être combinée avec non pas (plus, point) d'après le schéma non pas $P$, mais $Q$. Ce mais se trouve le plus souvent dans les dialogues conflictuels. L'assertion réfutée peut n'être pas explicitement attribuée ou attribuable à un énonciateur précis, différent ou non du locuteur $\mathrm{L}$, un effet polyphonique est, de toute façon, lisible. Mais réfutatif articule deux arguments antiorientés et surtout, il introduit un conflit de paroles. C'était le cas de l'exemple (44), où nous pouvons voir le mouvement argumentatif suivant :

- La proposition $\mathrm{P}$, sous-jacente à non-P (non point parce qu'il se serait dopé), est réfutée

- P est attribué à un énonciateur ou bien à une norme, un système de valeurs normé, culturel, idéologique, avec lequel le locuteur ne s'identifie pas

- Le passage de la réfutation (non-P) à l'assertion d'une proposition $Q$ (parce qu'il n'aurait pas avoué) jusqu'alors empêchée par la proposition $\mathrm{P}$ et qui valide la réfutation :

Parce qu'on a ce paradoxe, on a ce paradoxe, c'est que Richard Virenque, Pascal Hervé sont partie civile dans l'affaire et que sans cesse, mais sans cesse d'une manière parfois même méchante on on on met en cause Richard Virenque, non point parce qu'il se serait dopé mais parce qu'il n'aurait pas avoué alors qu'il affirme de s'être point doppé comme Pascal Hervé.

(Corpus 7/39)

Dans le corpus analysé, il y avait 12 exemples de mais de réfutation. 


\subsubsection{Mais phatique marquant les segments textuels}

Mais phatique marque le changement du point de vue. Ce mais est issu de la forme orale, décrite chez Ducrot (1980) dans Mais occupe-toi d'Amélie et peut porter sur l'énoncé précédent, mais encore plus souvent directement sur le contexte. On retrouve ce mais dans les dessins animés (exemple (45)) :

(45)

mais... mais ils détruisent et ils coulent mon bateau!

(Astérix : Le grand fossé, d'après Adam (1990)

Ce mais s'emploie surtout à l'oral et marque le changement du point de vue, une sorte d'opposition, le conflit entre ce qui est expecté et ce qui se passe en réalité. Il sert à marquer les morceaux de discours hétérogènes et perd ses valeurs habituelles. $75 \%$ d'occurrences de mais dans le corpus appartiennent à cette valeur.

Dans le discours analysé, nous l'avons retrouve sous les formes suivantes :

- mais phatique en énoncés déclaratifs

- mais phatique en énoncés interrogatifs

- mais phatique en énoncés exclamatifs

- mais phatique avec la valeur métalinguistique

- mais phatique en énoncés déclaratifs (exemple (46)) :

EP : Question Jacob, je vais compléter ce que j'ai souhaité dire. Je vais aussi signaliser à Madame Aubert : des produits de qualité. Mais tous les agriculteurs ne souhaitent que ça de faire des produits de qualité. Mais les produits de qualité ont un coût.

(Corpus 2/5)

Dans l'exemple (46), mais n'enchaîne pas sut le dit, mais introduit la réaction du locuteur qui sert à exprimer l'opposition à la situation. Dans ce cas, mais sert de la critique de l'énonciation du co-locuteur.

Mais dans les énoncés déclaratifs apparaît aussi en combinaison avec oui ou non (exemples (47) et (48)). En employant oui mais, le locuteur garde le contact avec le colocuteur, il se situe dans la co-énonciation. Avec non mais, le locuteur se place dans la rupture :

$P C: C$ 'est de la perversité là, quand même, oh, quand même, oui.

$F C$ : Oui, mais, je peux dire que j'ai entendu tellement de récits de ce type qu'ils vont bien au-delà de ce qu'ils en filment.

(Corpus 3/42)

JFM : Vous avez changé pour les transports aériens. Vous avez changé pour les télécommunications. Vous êtes en train de changer.. 
$B K$ : Non, mais non, mais non, je rejette tout.

(Corpus 7/35)

- mais phatique en énoncés interrogatifs n'est pas une demande d'information, mais une interrogation polémique avec la valeur argumentative de la proposition niée (exemple (49)) :

(49)

$C J$ : Mais quelles aides européennes sont données aux producteurs ... comme ça?

Précisez!

(Corpus 2/13)

- mais phatique dans l'exemple (50) introduit l'énoncé exclamatif qui marque la surprise:

BP : Mais c'est bien. Mais c'est très bien. Je vous félicite. Je n'ai jamais pu arriver si bien au sommet.

(Corpus 3/31)

- mais phatique dans le rôle métalinguistique

Ce même mais phatique sert à marquer métalinguistiquement le changement du point de vue ; il est typique pour l'oral. Sa fonction est de structurer les parties hétérogènes du discours apparaîssant aux niveaux différents, comme c'est le cas dans l'exemple (51). Mais porte ici sur l'énonciation, précisément sur le thème de l'émission.

(51)

$C J$ : Eh oui, je vais faire une proposition précise...

$J F C$ : Mais ce n'est pas la situation.

(Corpus 2/12)

\subsubsection{Mais concessif}

Mais concessif apparaît le plus souvent en combinaison avec la négation. Pourtant ce mais n'est pas paraphrasable avec non pas comme le mais réfutatif. Les propositions $\mathrm{P}$ et $\mathrm{Q}$ sont données comme contraires ; $\mathrm{P}$ implique non- $\mathrm{Q}$ et $\mathrm{Q}$ implique non-P. $\mathrm{P}$ et $\mathrm{Q}$ sont liées à des présuppositions concernant les idées reçues de notre civilisation, les topoï. Ce sont les exemples comme il est petit, mais fort (où l'idéé de la petitesse est contraire à l'idée de force). Mais concessif fait apparaître les valeurs pragmatiques sous-jacentes, laissant déviner les présuppositions du locuteur. La fonction du mais concessif est de renverser la présupposition (non-P $=>$ non- $Q$ ); nous pouvons soit le remplacer par et pourtant, soit le combiner avec pourtant :

Dans l'exemple (52) ci-dessous, mais est souligné par quand même :

$F C$ : et donc c'était cette idée de laisser quand même l'autre avoir un minimum de liberté. Mais au début quand même ceux qui se mariaient se disaient "tu es mon amour 
nécessaire", c'étaient ces amours nécessaires et contingents que distinguaient Sartre et de Beauvoir.

(Corpus 3/35)

\subsubsection{Mais argumentatif}

Mais argumentatif est la forme la plus complexe du fonctionnement de mais : selon J. M. Adam (1990 : 206), mais argumentatif dépend de l'espace discursif du locuteur. Mais argumentatif mobilise le carré argumentatif où $\mathrm{P}$ est argument pour la conclusion $C$ et $Q$ pour la conclusion non- $C$ et la conclusion non-C est plus forte : $C$ reste implicite. Un exemple de ce type est (53) où mais met en relation $P(j$ 'ai reconduit mon contrat avec Festina) et la conclusion qui a été donnée dans la question (ce n'est pas mon cas, je vais continuer à courir) et $\mathrm{Q}$ (mais aujourd'hui Richard va certainement mettre un terme à sa carrière) et la conclusion non-C (il ne va plus courir) :

MC : Est-ce que c'est le cas de Pascal Hervé? Non, apparement.

$P H:$ Non, $\downarrow$ moi j'ai reconduit mon contrat avec Festina mais aujourd'hui Richard va certainement mettre un terme à sa carrière. C'est quelque chose de très grave, non?

(Corpus 7/41)

L'analyse de mais nous surprend : comme il s'agissait de l'analyse des discours argumentatifs, nous pourrions constater que mais argumentatif va prévaloir dans le corpus analysé. Mais des 323 occurrences de mais dans le corpus, le plus fréquent était mais phatique avec 241 occurrences soit $75 \%$, dont 179 exemples du mais phatique dans les énoncés déclaratifs, 18 dans les énoncés interrogatifs, 18 dans les énoncés exclamatifs et 32 en emploi métalinguistique. Mais de renforcement était representé par 5 exemples soit $1 \%$, mais réfutatif de 12 exemples soit $4 \%$, mais concessif de 39 exemples soit $12 \%$ et mais argumentatif avec 26 exemples soit $8 \%$. Mais phatique a apparu aussi en combinaison avec les adverbes d'énonciation comme mais finalement, mais effectivement, mais justement, mais bien sûr.

\section{Conclusion}

Cette analyse a voulu démontrer l'utilisation des connecteurs dans le discours argumentatif oral à la base du corpus analysé. L'argumentation est tout le temps présente et véhiculée surtout par les connecteurs.

Mais, si on regarde d'abord la conclusion que nous avons tirée pour le connecteur mais, on se rend compte que la structure de mais argumentatif avec l'argument et deux conclusions n'est pas toujours respectée. Mais argumentatif est typique surtout pour les discours écrits. Dans le discours oral, le cadre argumentatif reste le même, mais véhicule les mêmes idées, pourtant toutes les étapes de l'argumentation ne sont pas 
prises en compte... L'argumentation dans le discours oral est ajoutée au niveau de l'énonciation et basée sur l'attitude envers le locuteur et gestion de l'énonciation dans la co-énonciation.

Les connecteurs à l'oral ont perdu leur valeur logique au sens strict du terme : ils en gardent les traces quelque part dans la conscience des hommes - et dans leur sens, si on peut le dire, mais à l'oral est déployée surtout leur valeur coénonciative de gestion de parole.

L'argumentation dans le discours oral se situe surtout au niveau interpersonnel de la gestion de la parole. Le discours, en tant qu'une négociation constante, est le lieu où le locuteur exprime soit son consensus, soit veut se démarquer par rapport à l'autre.

Alors, les connecteurs les plus fréquents à l'oral sont surtout marqueurs de structuration discursive et marqueurs de dimension interpersonnelle parmi les locuteurs.

\section{Litérature}

ADAM, J. M. (1990) : Eléments de linguistique textuelle. Bruxelles : Mardaga.

ANSCOMBRE, J. C. \& DuCRoT, O. (1983) : L'Argumentation dans la langue. Bruxelles : Mardaga.

BERTHOUD, A.-C. (1996) : Paroles à propos: approche énonciative et interactive du topic. Paris : Ophrys.

vaN DiJk, T. A. (1981): Studies in the Pragmatics of Discourse. The Hague, Paris, New York: Mouton Publishers.

DK, S. C. (1989): The Theory of Functional Grammar, Part I: The structure of the clause. Dordrecht: Foris.

Dik, S. C. (1997): The Theory of Functional Grammar, Part II: Complex and derived constructions. ed. Kees Hengeveld, Berlin, New York: Mouton de Gruyter.

Ducror, O. et al., (1980) : Les mots du discours. Paris : Minuit.

GERECHT, A. M. (1987) : Alors, connecteur temporel, connecteur argumentatif et marqueur du discours, dans : Cahiers de linguistique française 8. Genève, p. 69-79

HARRIS, Z. S. (1970): Papers in Structural and Transformational Linguistics. Dordrecht: Reidel.

HyBERTIE, C. (1996) : La conséquence en français, Paris : Ophrys.

MOESCHLER, J. (1985) : Argumentation et conversation. Pariz : Hâtier.

MOESCHLER, J. et al. (1994) : Language et pertinence. Nancy : PUN. Coll. Processus discursifs.

Morel, M. A. (1996a) : La concession. Paris : Ophrys.

Morel, M. A., Danon-BollEAU, L. (1998a) : La grammaire de l'intonation : Exemple du français. Paris-Gap : Ophrys.

Reboul, A. \& Moeschler, J. (1998) : Pragmatique du discours. Pariz : Armand Collin.

RIEGEL, M., PEllat, J. C., Rioul, R. (1994) : Grammaire methodique du francais. Paris : PUF.

RoULET, E. \& al. (1985) : L'Articulation du discours en français contemporain. Bern : Lang.

SCHLAMBERGER BREZAR, M. (2000) : Les connecteurs en combinaison avec les marqueurs modaux : l'exemple du français et du slovène. Linguistica XL/2, 123-135.

Traverso, V. (1998) : La conversation familière. Lyon : Presses universitaires de Lyon. 


\section{SKLADENJSKI IN PRAGMATIC̆NI VIDIKI POVEZOVALCEV V FRANCOSKIH ARGUMENTACIJSKIH BESEDILIH}

Zanimanje za konektorje-povezovalce se je začelo v sedemdesetih letih in je ostalo prisotno v različnih vejah lingvistike do danes. Povezovalci so danes večinoma analizirani v okviru pragmatike, saj njihov domet presega stavčno skladnjo; navezujejo se lahko na prejšnje izreke, na izrekanje in celo na sam kontekst.

Po rezultatih analize francoskih argumentacijskih besedil, ki je potekala na šest urah posnetih in zapisanih besedil francoskih okroglih miz "Bouillon de culture" in "Polémiques" so bili najpogostejši povezovalci et, parce que, alors, donc, mais.

Pokazalo se je, da v govorjenem diskurzu prevladujejo povezovalci, ki se oddaljujejo od osnovne logične sheme, ki jo imajo kot vezniki. Predvsem to velja za mais. Logična shema je še prisotna $\mathrm{v}$ zavesti govorca, povezovalci pa predvsem delujejo kot zaznamovalci zgradbe diskurza in urejajo medsebojne odnose med govorcema v okviru soizrekanjske sheme. 